Massimo Trappolini

Alessandra Clarice

Alfredo Scorza

Lucia Angrisani

Federica Trappolini

Massimiliano Rocchietti March

Maria Proietta

\section{A case of spontaneous intracranial hypotension with typical magnetic resonance images}

Received: 19 August 2005

Accepted in revised form: 24 September 2005

Published online: 10 November 2005

M. Trappolini • A. Clarice • A. Scorza

L. Angrisani • F. Trappolini

M. Rocchietti March • M. Proietta

Department of Internal Medicine,

Director Prof. G. Aliberti,

S. Andrea Hospital, II Medicine School,

University of Rome "La Sapienza",

Via di Grottarossa 1035/1039,

Rome, Italy

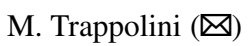

Via Filippo Cordova 36,

I-00139 Rome, Italy

e-mail: massimo.trappolini@uniroma1.it

Tel.: +39-0680-345042

Fax: +39-0680-345074

\begin{abstract}
Spontaneous intracranial
hypotension $(\mathrm{SIH})$ is a rare syndrome defined by postural headache, associated with a low cerebrospinal fluid pressure, without history of previous dural trauma or invasive treatment on rachis. We reported a case of a patient with postural headache caused by SIH identified by magnetic resonance images and treated with saline solution infusion with complete remission of symptoms.
\end{abstract}

Keywords Spontaneous intracranial hypotension - Postural headache • Pachymeningeal enhancement • Hygroma

\section{Introduction}

Spontaneous intracranial hypotension $(\mathrm{SIH})$ is a rare syndrome, characterized by a postural headache associated with a low cerebrospinal fluid (CSF) pressure and with no history of previous dural trauma or invasive treatment on rachis [1]. SIH is more frequent in women than men and often self-limiting [2]. A postural headache is one that occurs or worsens in less than $15 \mathrm{~min}$ after assuming an upright position and disappears or improves in less than 30 min after resuming the recumbent position [3].

We present a patient with postural headache and with magnetic resonance imaging (MRI) typical of SIH.

\section{Case report}

A 48-year-old female, with a history of mild hypertension well treated with ACE inhibitor was admitted to our department for persistent fronto-occipital headache, nausea, dizziness, nystagmus and photophobia. Cervicobrachial and interscapular pain lasted for $15 \mathrm{~min}$ and could only be relieved upon lying down. The headache showed postural variation, worsening when she was in an orthostatic position and improving when she got into a recumbent position. Neither fever nor neck rigidity could be found. These symptoms had arisen seven days before admission to the hospital. 
She took steroidal anti-inflammatory drugs without advantage. The patient's medical history was negative for trauma, surgical treatment and/or invasive treatment on the rachis or on the central nervous system.

On admission, her physical and neurologic examination findings were normal (no neck rigidity could be found), as well as the findings of electrocardiogram, chest radiography, electroencephalograph, cranial computed

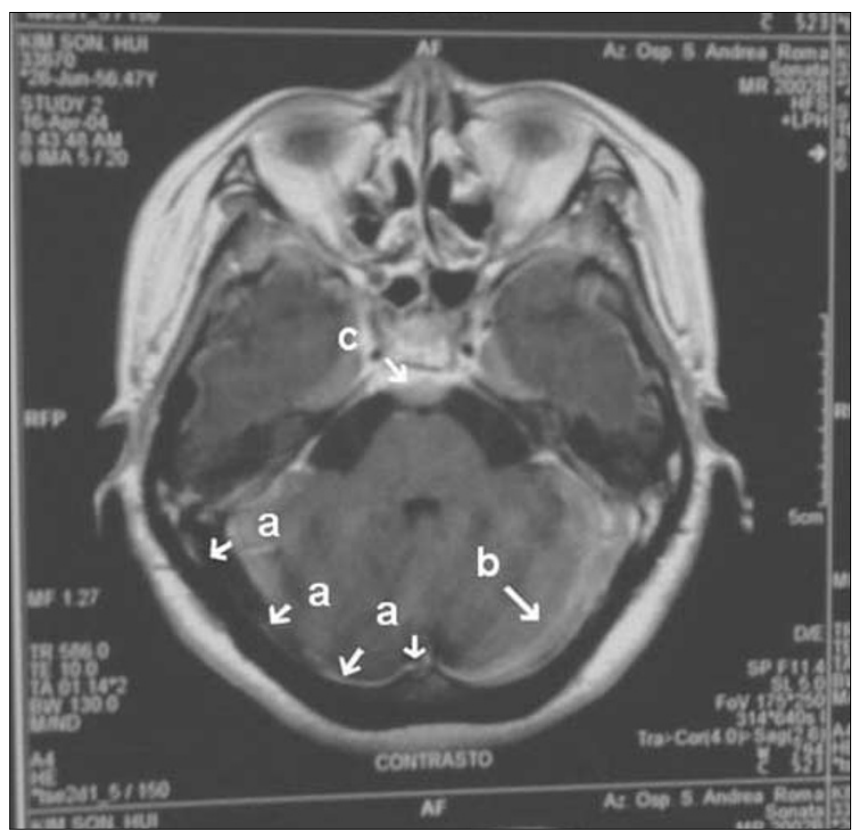

Fig. 1 Magnetic resonance imaging in spontaneous intracranial hypotension: (a) pachymeningeal enhancement, (b) subdural fluid collection (aka hygroma), (c) subdural haematoma

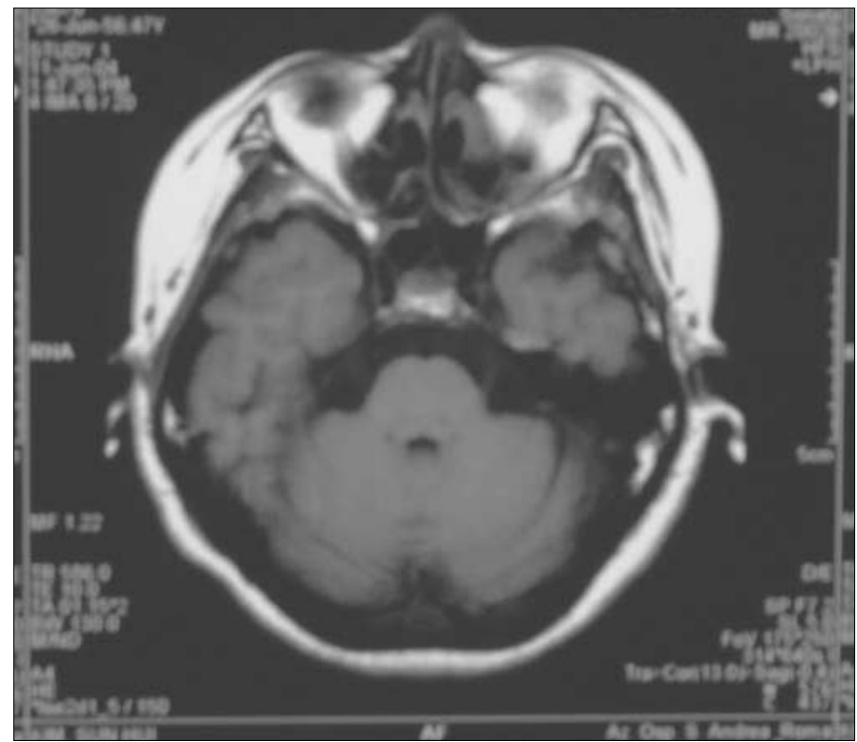

Fig. 2 Magnetic resonance imaging post-treatment (after 45 days). Resolution of typical signs demonstrated before tomography (CT) and routine laboratory studies, except for hypertriglyceridaemia. Cervical radiography showed degenerative spinal discopathy (C5-C6).

The patient was treated with corticosteroids, pyridol and amitriptyline. As her symptoms did not regress, a brain and medulla MRI with gadolinium was necessary, showing: "Pachymeningeal supratentorial gadolinium enhancement, subdural fluid collections without mass effect" (Fig. 1).

In consideration of the symptoms and MRI we diagnosed "Headache from SIH".

The patient was treated with saline infusion i.v. and bed rest, and her symptoms improved greatly over a period of one week. At discharge she was asymptomatic.

Two months later, a brain MRI showed the complete regression of the pachymeningeal enhancement and subdural fluid collections that showed before (Fig. 2). Twelve months later she continues to do well and she remains headache free.

\section{Discussion}

SIH is a rare event but has an increasing tendency $[4,5]$. Orthostatic headache is the principal manifestation of $\mathrm{SIH}$, associated with low CSF pressure. Other associated symptoms are nausea, emesis, blurred vision, diplopia, tinnitus and change in hearing. These symptoms show a typical clinical picture that could be misdiagnosed, causing a significant delay in the onset of treatment [5].

A consequence of CSF volume depletion is descent of the brain. This leads to traction or distortion of various anchoring pain-sensitive structures of the brain, causing orthostatic or primary headaches. Dilatation of cerebral veins and venous sinuses may also play a role in the production of these headaches [1].

It is possible that in some patients a combination of several factors, such as connective tissue disorders, dural sac abnormalities or Marfan syndrome [4], may predispose for SIH, but not in our patient. In many patients it is not possible to identify the cause of SIH [1].

SIH must be suspected in cases of postural headache. Diagnostic procedures (CT, MRI) or invasive techniques (lumbar puncture or radionucleotide cisternography) allow diagnosis of SIH. TC is usually normal, as in our case, and is useless for diagnosis [2]. Occasionally CT revealed subdural fluid collections or increased tentorial enhancement.

Gadolinium MRI shows distinct abnormalities of SIH [6]. The three most characteristic MRI features of SIH are enhancement of the pachymeningeal, downward displacement of brain and subdural fluid collection $[5,6]$.

Dural puncture is an invasive procedure and it can show undiagnostic data [1]. CSF pressure is typically very 
low. Sometimes, especially in high-flow leaks, it may be unmeasurable, or occasionally even negative or normal. The CSF cell count also shows considerable variability; cytology and microbiology is always normal and CSF glucose is never low. Fluid examination can also be variable: the fluid itself is typically clear and colourless, but sometimes may be xanthochromic.

Indium-111 radioisotope cisternography is the most sensitive and specific examination for documenting the leakage of CSF and for diagnosis of SIH [7], but it is an invasive procedure and, above all, it is not available in every hospital. It should be restricted to patients who do not improve with conservative treatment or when MRI is not diagnostic, because in these cases it is essential to begin more specific treatments.

In our case we suspected SIH because of symptoms and the diagnosis was confirmed by MRI. Moreover, conservative therapy with saline solution infusion determined the complete remission of symptoms. This outcome has added to the diagnosis of SIH. For this rea- son we have not carried out more invasive diagnostic procedures.

Besides hydration or overhydration, caffeine and theophylline have been demonstrated to be effective in some studies, but their effectiveness is often unimpressive and of doubtful durability. The efficacy of steroids has not been proved and is mostly anecdotal [1].

In patients in which an initial trial of conservative management fails, intrathecal fluid infusion or epidural infusion of crystalloids or colloids may be tried [8]. Nevertheless, when the point of CSF fistula is known, the treatment of choice is epidural blood patch or percutaneous placement of fibrin sealant $[9,10]$.

In summary, SIH is a rare syndrome and the typical postural headache, without trauma or invasive treatment on rachis, is suggestive of $\mathrm{SIH}$, but only MRI findings can indicate the correct diagnosis. In fact, MRI is the non-invasive diagnostic gold standard with demonstration of typical signs. Therefore it permits a conservative treatment to be commenced early, with the complete remission of symptoms.

\section{References}

1. Mokri B (2003) Headaches caused by decreased intracranial pressure: diagnosis and management. Curr Opin Neurol 16:319-326

2. Bachmann-Mennenga B, Philipps J, Haukamp F, Reinbold WD (2004) Spontaneous intracranial hypotension. A rare syndrome with good treatment options. Anaesthesist 53:45-52

3. Headache Classification Subcommittee of the International Headache Society (2004) The International Classification of Headache Disorders, 2nd edn. Cephalalgia 24[Suppl 1]:9-160
4. Schievink WI, Gordon OK, Tourje J (2004) Connective tissue disorders with spontaneous spinal cerebrospinal fluid leaks and intracranial hypotension: a prospective study. Neurosurgery 54:65-70

5. Mokri B (2004) Spontaneous low cerebrospinal pressure/volume headaches. Curr Neurol Neurosci Rep 4:117-124

6. de Noronha RJ, Sharrack B, Hadjivassiliou M, Romanowski CA (2003) Subdural haematoma: a potentially serious consequence of spontaneous intracranial hypotension. $\mathrm{J}$ Neurol Neurosurg Psychiatry 74:752-755
7. Bai J, Yokoyama K, Kinuya S, Konishi S, Michigishi T, Tonami N (2002) Radionuclide cisternography in intracranial hypotension syndrome. Ann Nucl Med 16:75-78

8. Aldrete JA (1994) Persistent postdural-puncture headache treated with epidural infusion of dextran. Headache 34:265-267

9. Crul BJ, Gerritse BM, Van Dongen RT, Schoonderwaldt HC (1999) Epidural fibrin glue injection stops persistent postdural puncture headache. Anesthesiology 91:576-577

10. Gaukroger PB, Brownridge P (1987) Epidural blood patch in the treatment of spontaneous low CSF pressure headache. Pain 29:119-122 